\title{
The Modulatory Effects of Vitamin D on the Expression of IL-12 and TGF- $\beta$ in the Spinal Cord and Serum of Mice with Experimental Autoimmune Encephalomyelitis
}

\author{
Rayhaneh Ahangar-Parvin ${ }^{1,2}$, Marzieyeh Mohammadi-Kordkhayli ${ }^{3}$, Sayyed Vahab Azizi ${ }^{1,2}$, Maryam \\ Nemati $^{4,5}$, Hossain Khorramdelazad ${ }^{1,2}$, Zahra Taghipour ${ }^{6}$, Zuhair Hassan ${ }^{7}$, Seyed Mohammad \\ Moazzeni $^{7}$, Abdollah Jafarzadeh ${ }^{1,2 *}$ \\ 1. Molecular Medicine Research Center, Rafsanjan University of Medical Sciences, Rafsanjan, Iran \\ 2. Dept. of Immunology, Medical School, Rafsanjan University of Medical Sciences, Rafsanjan, Iran \\ 3. Dept. of Immunology, School of Public Health, Tehran University of Medical Sciences, Tehran, Iran \\ 4. Dept. of Laboratory Sciences, Para-Medical School, Kerman University of Medical Sciences, Kerman, Iran \\ 5. Dept. of Immunology, Medical School, Kerman University of Medical Sciences, Kerman, Iran \\ 6. Dept. of Histology, Medical School, Rafsanjan University of Medical Sciences, Rafsanjan, Iran \\ 7. Dept. of Immunology, Medical School, Tarbiat Modares University, Tehran, Iran
}

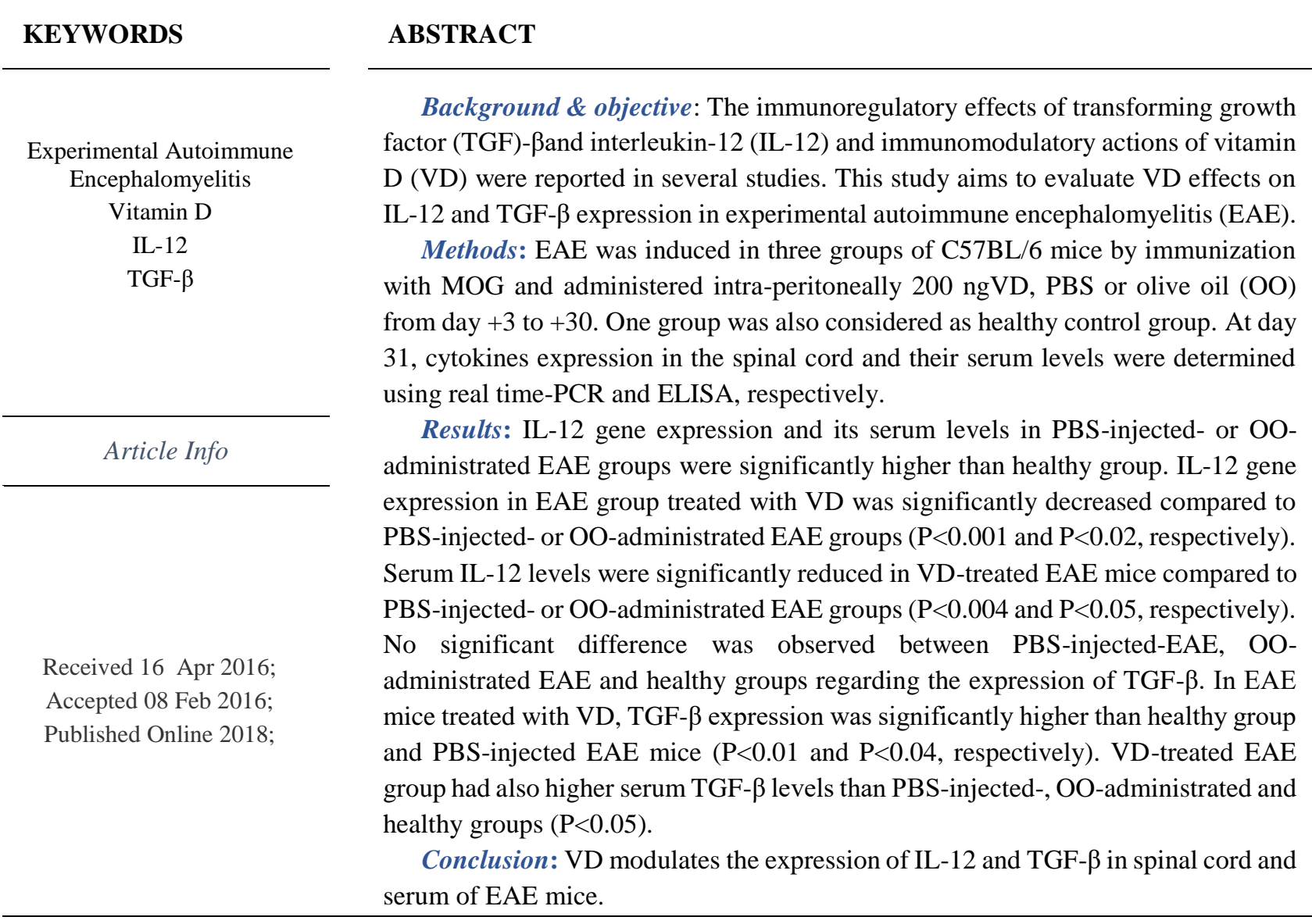

Corresponding information: Abdollah Jafarzadeh, Professor of Immunology, Department of Immunology, Medical School, Rafsanjan University of Medical Sciences, Rafsanjan, Iran.Tel:+98 343433 9042, Fax:+98 343433 9660, E-mail: Jafarzadeh@kmu.ac.ir

Copyright $\odot$ 2017, IRANIAN JOURNAL OF PATHOLOGY. This is an open-access article distributed under the terms of the Creative Commons Attribution-noncommercial 4.0 International License which permits copy and redistribute the material just in noncommercial usages, provided the original work is properly cited.

\section{Introduction}

Multiple sclerosis (MS) is a chronic autoimmune response-mediated inflammatory disease of the central nervous system (CNS), which results in neuronal demyelination and axonal degeneration
(1). The experimental model of MS disease is named autoimmune encephalomyelitis (EAE), which is inducible in susceptible animals by immunization with myelin-derived components 
such as myelin basic protein (MPB), proteolipid protein (PLP) or myelin oligodendrocyte protein (MOG) mixed with a suitable adjuvant (2).

Rupture of the blood-brain barrier (BBB) and migration of activated leukocytes are among the earliest defects that occur in MS and EAE diseases (3). Dendritic cells (DCs) migrate across the BBB and induce differentiation of naïve myelin specific naïve $\mathrm{CD} 4^{+} \mathrm{T}$ cells into pathogenic Th1 and Th17 that may be accountable for demyelination in MS and $\operatorname{EAE}(3,4)$, while Treg cells may confer protection against diseases and the Th2 role remains controversial $(4,5)$. Increased levels of a Th17 cell-related chemokine C-C motif ligand 20 (CCL20) and diminished levels of a Th2/Treg cellrelated chemokine $\mathrm{C}-\mathrm{C}$ motif ligand 22 (CCL22) were also observed in patients with MS $(6,7)$.

Structurally, IL-12 is composed of two covalently linked subunits, including P35 and P40 which is produced mainly by antigen-presenting cells (APCs), including macrophages and DCs (8). The P35 subunit is unique to IL-12, whereas P40 subunit is shared with IL-23, another proinflammatory cytokine required for the establishment of Th17 cell-related immune responses (9). Mice with a P35 knockout (KO) cannot develop Th1 cell-related immunity, while those with a P40 KO unable to develop both Th1and Th17 cells-related immunity $(9,10)$. The P40 subunit also induces the expression of IL-16 (a lymphocyte chemoattractant factor), nitric oxide synthase (NOS) and tumor necrosis factor- $\alpha$ (TNF$\alpha)$ in microglia and macrophages and promotes the migration of macrophages and dendritic cells (11, 12).

IL-12 receptor is expressed on several immune cells, including natural killer (NK) cells, DCs, T and $\mathrm{B}$ cells $(8,13)$. IL-12 is a pro-inflammatory and its major functions include enhancing the cytotoxic activity of $\mathrm{CD}^{+} \mathrm{T}$ lymphocytes and NK cells, increasing the production of IFN- $\gamma$ from NK and T cells, inducing the differentiation of naïve $\mathrm{CD}^{+}$ Th0 cells into the Th1 cells and increasing antibody-dependent cellular cytotoxicity (ADCC) against tumor cells $(8,14)$.

Transforming growth factor (TGF)- $\beta$ is an important pleiotropic cytokine with powerful immunomodulatory effects which is released by regulatory $\mathrm{T}$ (Treg) cells (15). Different functions have been attributed to TGF- $\beta$ such as stimulating Treg cells differentiation, enhancing IL-10 secretion by macrophages, inducing the generation of tolerogenic DC, reducing IL-1, IL-12, TNF- $\alpha$ and TNF- $\beta$ production by APCs, modulating the toll-like receptor 4 (TLR4) expression, decreasing the macrophage and microglia functions and suppressing the development of Th1- and Th2 lymphocytes $(15,16)$.

TGF- $\beta$ plays a key role in maintaining tolerance by controlling proliferation and differentiation of the effector lymphocytes $(15,16)$. There are several investigations showing that TGF- $\beta$ confer protection against MS and EAE diseases. TGF- $\beta$ ameliorates EAE and inhibits the entrance of sensitized T cells into the CNS (17). Treatment of EAE mice with an antibody against TGF- $\beta$ worsens EAE severity (16).

Vitamin D has various immunoregulatory and anti-inflammatory characteristics (18). Some beneficial effects of treatment of EAE mice with vitamin $\mathrm{D}$ including attenuation of the disease severity, delaying in disease beginning and low aggregation of the inflammatory cell into the CNS were observed in our previous study (19). Longterm medication with anti-inflammatory drugs may have deleterious side effects. Therefore, novel efficient therapeutic programs with fewer side effects should be considered for the treatment of inflammatory diseases. This study aimed to evaluate the effects of vitamin $\mathrm{D}$ on the expression of IL- 12 and TGF- $\beta$ in the spinal cord and serum of C57BL/6 mice subjected to EAE induction by immunization with a myelin-derived antigen named MOG.

\section{Materials and Methods}

\section{Mice}

The female C57BL/6 mice (6-8 weeks old) were obtained from the Pasteur Institute (Tehran, Iran) and housed in standard controlled conditions; temperature $20 \pm 2^{\circ} \mathrm{C}$ and a 12 -hour light/12-hour dark cycle with ad libitum access to mouse nutrition and water in a standard laboratory manner. To avoid any stress reaction possibly caused by novel environmental cues, the research protocol was 
performed in a room where the mice were housed. All experiments were performed in accordance with the Ethics Committee on Animal Experimentation of Rafsanjan University of Medical Sciences (IR.RUMS.REC.1394.275) and were also in agreement with the National Research Council Guide (NRC, 2011).

\section{Induction and scoring of EAE}

EAE was induced by immunization with a $\mathrm{MOG}_{35-55}$ peptide as explained previously (20). The MOG is a component of the myelin sheath of nerves and is known as a major target autoantigen in MS and EAE diseases. Briefly, the C57BL/6 mice were immunized by subcutaneous (s.c) delivery in two places in the flank on day 0 with injecting $400 \mu \mathrm{g}$ of $\mathrm{MOG}_{35-55}$ peptide (Alexis, Switzerland) mixed in complete Freund's adjuvant comprising $5 \mathrm{mg} / \mathrm{mL}$ of Mycobacterium tuberculosis. The mice received two additional injections of $250 \mathrm{ng}$ of pertussis toxin intraperitoneally (i.p) on days 0 and 48 hours after immunization with MOG. The EAE clinical score was measured using a standardized scoring criteria (21) as follows; 0, no signs (no abnormality); 1, loss of tail tone; 2, flaccid tail; 3 , one hind limb paralysis; 4, two hind limbs paralysis; 5, paralysis of forelimb and hind limb; 6, dead animal. Paralyzed mice had comfortable access to water and nutrition.

\section{Experimental design}

Four groups of female C57BL/6 mice (6 mice in each group) were enrolled into the study. Group I was considered as a healthy control group. The mice in group I were not immunized with MOG and treated with PBS. The mice in groups II, III and IV were subjected to EAE induction and treated with PBS, olive oil (as a vitamin D vehicle) or $200 \mathrm{ng}$ of vitamin D (22). Groups II, III and IV were considered as PBS-injected EAE group, olive oiladministrated EAE group and vitamin D-treated EAE group, respectively.

The 1, 25 dihydroxy vitamin D3 (1,25(OH)2D3) was obtained from Osveh company (Tehran, Iran). For induction of EAE, mice were immunized on the first day by a MOG peptide mixed with complete Freund adjuvant containing killed Mycobacterium tuberculosis. From day +3 to +30 , the mice were intraperitoneally (i.p) treated with $100 \mu \mathrm{L}$ PBS or olive oil (as a vitamin D vehicle) in control groups and $200 \mathrm{ng}$ vitamin D in the same volume of olive oil (every two days) in vitamin D-treated group. The mice were scored daily for exhibition of clinical symptoms of EAE, from the day of MOG injection (day 0) until the day 30. Mice were killed on day 31 and their spinal cords removed for RNA extraction and gene expression analyses.

\section{RNA extraction, reverse transcription and quantitative real-time PCR}

Quantitative real time-PCR method was used to determine gene expression of the IL- 12 and TGF- $\beta$ in the spinal cords. Total RNA was extracted from the spinal cords using Trizol Reagent (purchased from Invitrogen, Carlsbad, CA) according to the manufacturer's guideline. Electrophoresis on the agarose gel pre-treated with ethidium bromide was used to determine the quality of extracted RNA. A spectrophotometer system was used to determine RNA purity based on measuring absorption at 260 $\mathrm{nm}$ and $280 \mathrm{~nm}$ and determination of 260/280 ratio.

A complementary DNA (cDNA) synthesis kit (Bionner, Korea) that contained both oligo (dT) and random hexamer primers was used to convert the extracted RNA to the cDNA. The reverse transcription amplification protocol included: $70^{\circ} \mathrm{C}$ for $10 \mathrm{~min}$ (in the absence of reverse transcription enzyme), $20^{\circ} \mathrm{C}$ for $1 \mathrm{~min}$ (cooling stage), addition of reverse transcription enzyme, $42^{\circ} \mathrm{C}$ for $60 \mathrm{~min}$ and finally the protocol was completed by a step at $95^{\circ} \mathrm{C}$ for $10 \mathrm{~min}$ to terminate the activation of reverse transcription enzyme.

Real-time PCR protocol was performed using a Bio-Rad CFX96 system (Bio-Rad, USA) in a triplicate manner using a SYBR green master mix (Bionner, Korea), mixed with $200 \mathrm{ng}$ of template cDNA with $2 \mu \mathrm{L}$ suitable primers (10 pmol stock). Sequences of the used primers and the sizes of amplification products are demonstrated in Table 1 . 
Table 1. Used Primers for mRNA Expression of IL-12and TGF- $\beta$ in the Spinal Cords

\begin{tabular}{|c|c|c|}
\hline Gene & Primer & Amplification product (bp) \\
\hline \multirow{2}{*}{ IL-12 (P35) } & Forward: CCACCСТTGCССТCСТАAAC & \multirow{2}{*}{132} \\
\hline & Reverse: GTTTTTCTCTGGCCGTCTTCA & \\
\hline \multirow{2}{*}{ IL-12 (P40) } & Forward: GGAAGCACGGCAGCAGAATA & \multirow{2}{*}{180} \\
\hline & Reverse: AACTTGAGGGAGAAGTAGGAATGG & \\
\hline \multirow{2}{*}{ TGF- $\beta$} & Forward: ATTCCTGGCGTTACCTTG & \multirow{2}{*}{114} \\
\hline & Reverse: GTATTCCGTCTCCTTGGTTC & \\
\hline \multirow{2}{*}{$\beta$-Actin } & Forward: AGAGGGAAATCGTGCGTGAC & \multirow{2}{*}{138} \\
\hline & Reverse: CAATAGTGATGACCTGGCCGT & \\
\hline
\end{tabular}

The thermal cycling program was as follows; an initial heating at $95^{\circ} \mathrm{C}$ for $15 \mathrm{~min}, 40$ cycles of $95^{\circ} \mathrm{C}$ for $30 \mathrm{~s}$ and $60^{\circ} \mathrm{C}$ for $30 \mathrm{~s}$ and eventually $72^{\circ} \mathrm{C}$ for 30 s. Primers were synthesized by the Bionner Company (Korea). For normalization of the amplified cytokine target genes, the $\beta$-actin gene was used as an internal control or housekeeping gene. The amount of IL-12 and TGF-B expression in the spinal cords expressed as units relative to the amount of $\beta$-actin expression calculated by the 2 $\Delta \Delta \mathrm{Ct}$ formula. The melting curves and data processing were performed using CFX manager software version 1.1.308.111 (Bio-Rad, USA). The PCR products were also electrophoresed and visualized on a $1 \%$ agarose gel comprising 0.5 $\mathrm{mg} / \mathrm{mL}$ ethidium bromide (Figure 1).

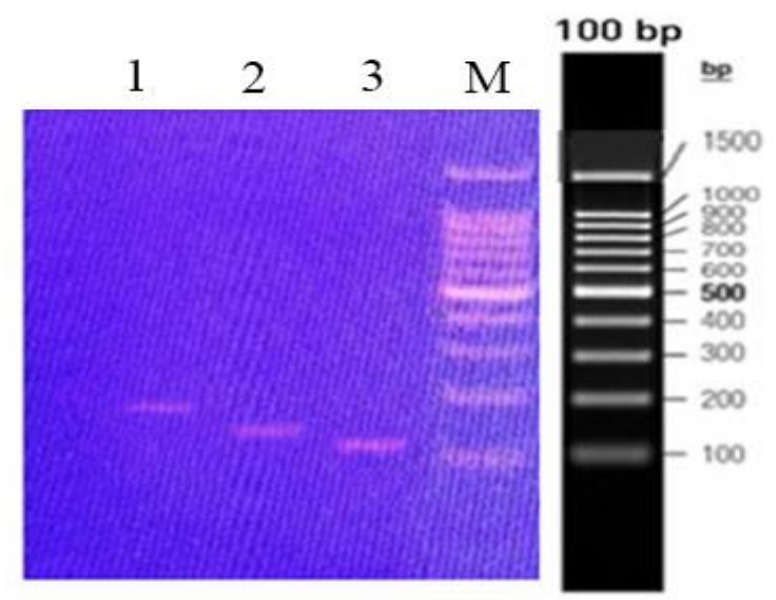

Figure 1. PCR amplification of IL-12 and TGF- $\beta$ genes. Columns 1, 2 and 3 show the PCR products of IL-12P40, IL-12P35 and TGF- $\beta$ from a healthy normal mouse, respectively.

Column M shows the DNA Marker (ladder).

\section{Measurement of Serum IL-12 and TGF- $\beta$} Levels

As mentioned, the mice were killed on day 31 and blood collection was performed through cardiac puncture. Then the serum specimen were separated and placed at $-20^{\circ} \mathrm{C}$ until analysis. Serum IL-12 and TGF- $\beta$ concentrations were quantitated using commercial enzyme-linked immunosorbent assay (ELISA) kits (eBioscience, UK). The sensitivity degrees were 4.0 and $15.0 \mathrm{Pg} / \mathrm{mL}$ for IL12 and TGF- $\beta$ kits, respectively.

\section{Statistical analysis}

Data was expressed as mean \pm SEM. Statistical comparison was analyzed by a computer program (SPSS version 18, Chicago, IL, USA) using ANOVA and Student's $t$ test, as appropriate. The differences were considered significant, when the $\mathrm{P}$ values were less than 0.05 .

\section{Results}

\section{Vitamin D effects on EAE clinical symptoms}

The EAE clinical scores in vitamin D-treated mice and control groups are demonstrated in Figure 2. Clinical signs of EAE appeared in the PBSinjected EAE mice and olive oil-administrated EAE group on days 10 and 9, respectively. The vitamin D-treated EAE group exhibited clinical signs of EAE later on day 13. The maximum mean clinical score (MMCS) was $5 \pm 0.00$ for PBS-injected EAE mice, $4.5 \pm 0.28$ for olive oil-administrated EAE group and $2.40 \pm 0.40$ for EAE mice received vitamin D treatment. The MMCS in EAE group receiving vitamin $\mathrm{D}$ treatment was lower than PBSinjected EAE mice and olive-administrated EAE group ( $\mathrm{P}<0.001$ using student's $t$ test). 


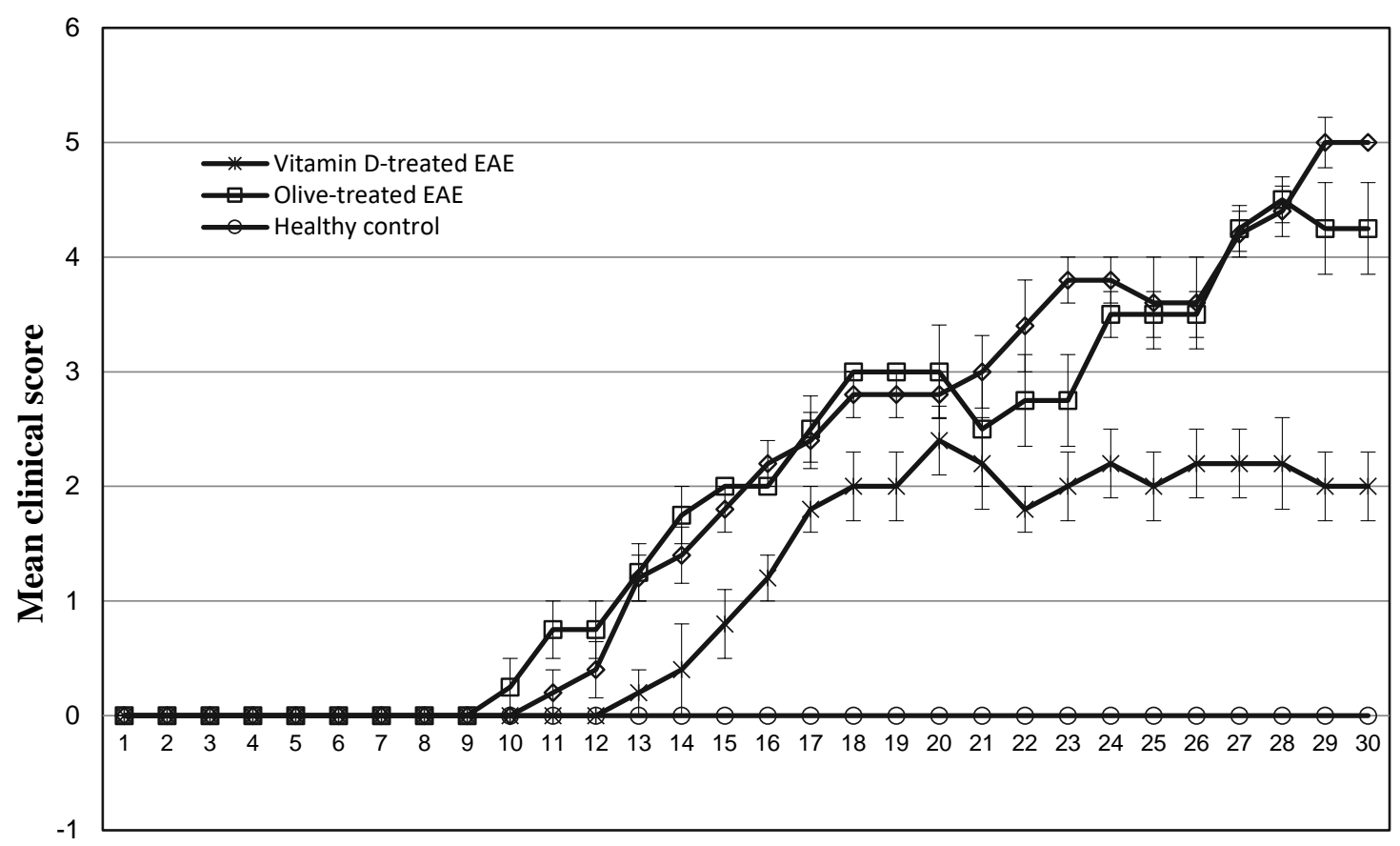

Time of post MOG immunization (days)

Fig 2. Comparison of the clinical scores of the EAE between vitamin D-treated and control groups.

The maximum mean clinical score was significantly lower in vitamin D -treated EAE groups as compared with PBSor olive treated EAE mice $(\mathrm{P}<0.01$ and $\mathrm{P}<0.02$, respectively; by using student's $t$ test).

The effects of vitamin $D$ on the gene expression of IL-12 in spinal cords

PBS-injected EAE mice had a higher mRNA expression of IL-12 P35 and IL-12 P40 than the healthy group $(\mathrm{P}<0.001)$ (Table 2, Figures $3 \& 4)$.

mRNA expression of IL-12 P35 and IL-12 P40 in olive oil-administrated EAE group were also significantly higher than the healthy group $(\mathrm{P}<0.001$ and $\mathrm{P}<0.04$, respectively). mRNA expression of IL-12 P35 and IL-12 P40 in EAE group receiving vitamin $\mathrm{D}$ treatment were significantly lower than PBS-injected EAE mice and olive oil-administrated EAE group $(\mathrm{P}<0.001$ and $\mathrm{P}<0.02$, respectively). mRNA expression of IL12 P35 and IL-12 P40 in olive oil-administrated group was also significantly lower than in PBSinjected EAE group $(\mathrm{P}<0.002)$ (Table 2, Figures 3 \& 4).

Table 2. mRNA Expression of IL-12 and TGF- $\beta$ in Spinal Cords and Their Serum Levels in Vitamin D-treated EAE Mice and Control Groups

\begin{tabular}{cccccc}
\hline \multirow{2}{*}{ Groups } & \multicolumn{3}{c}{ In spinal cord (Fold) } & \multicolumn{2}{c}{ In serum $($ Pg/mL) } \\
\cline { 2 - 5 } & IL-12 P35 & IL-12 P40 & TGF- $\beta$ & IL-12 & TGF- $\beta$ \\
\hline Healthy group & $0.98 \pm 0.16$ & $0.99 \pm 0.26$ & $1.00 \pm 0.19$ & $14.02 \pm 1.05$ & $7.40 \pm 1.35$ \\
\hline PBS-injected EAE & $64.58 \pm 9.36$ & $32.87 \pm 5.13$ & $1.39 \pm 0.08$ & $36.00 \pm 2.14$ & $8.13 \pm 1.69$ \\
\hline Olive oil-treated EAE & $13.80 \pm 2.63$ & $4.00 \pm 0.99$ & $1.72 \pm 0.40$ & $31.27 \pm 3.96$ & $8.15 \pm 2.86$ \\
\hline vitamin D-treated EAE & $4.61 \pm 1.72$ & $0.71 \pm 0.24$ & $2.90 \pm 0.92$ & $20.08 \pm 2.94$ & $15.29 \pm 3.37$ \\
\hline
\end{tabular}




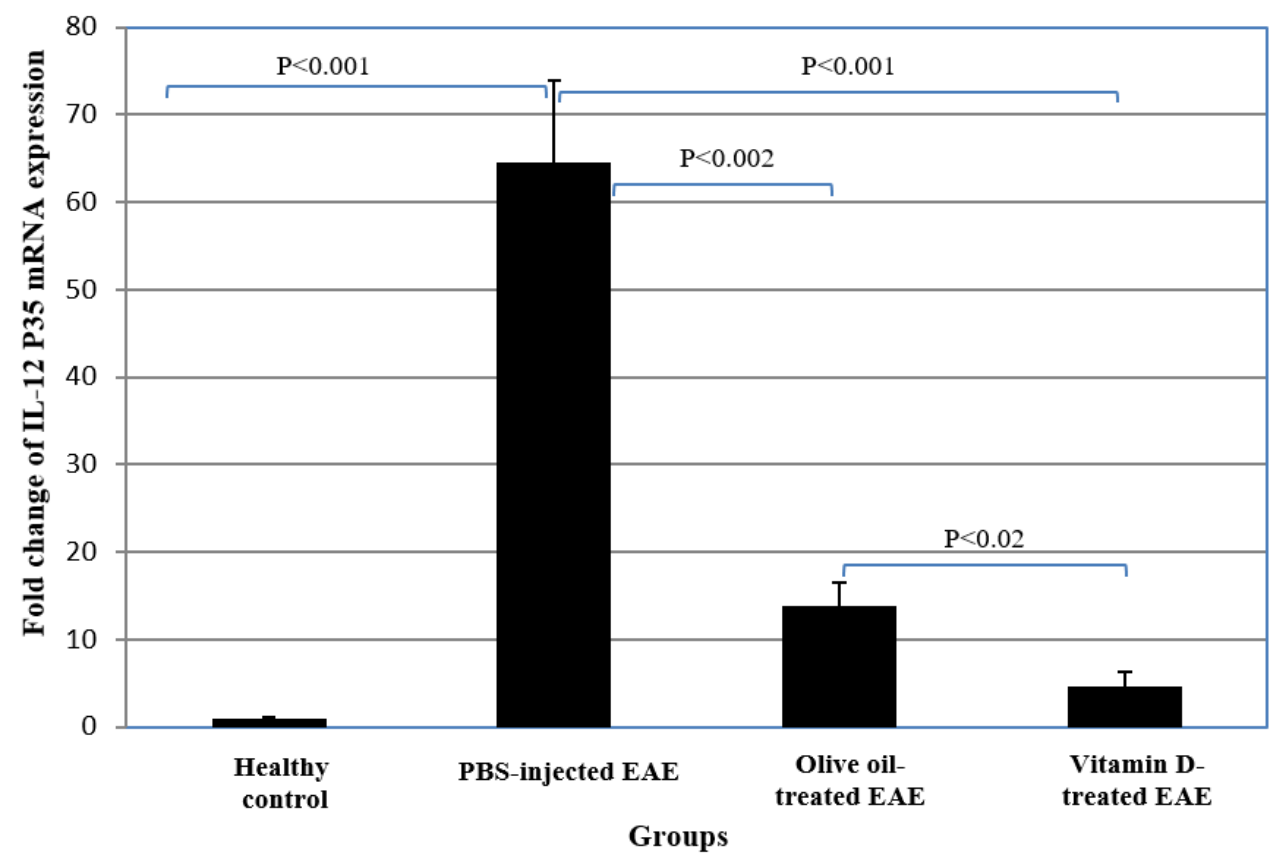

Fig 3. Comparison of the expression of IL-12 P35 mRNA between vitamin D-treated and control groups. The mRNA expression of the IL-12 P35 in PBS-treated EAE group was significantly higher than in the healthy group $(P<0.001$, by using student's $t$ test). The expression of IL-12 P35 mRNA in both vitamin D-treated EAE mice and olive oil-treated EAE group were decreased as compared with PBS-treated EAE group $(P<0.001$ and $P<0.002$, respectively; by using student's $t$ test). The expression of IL-12 P35 mRNA in vitamin D-treated EAE group was also significantly lower than olive oil-treated EAE group $(P<0.02$, by using student's $t$ test $)$.

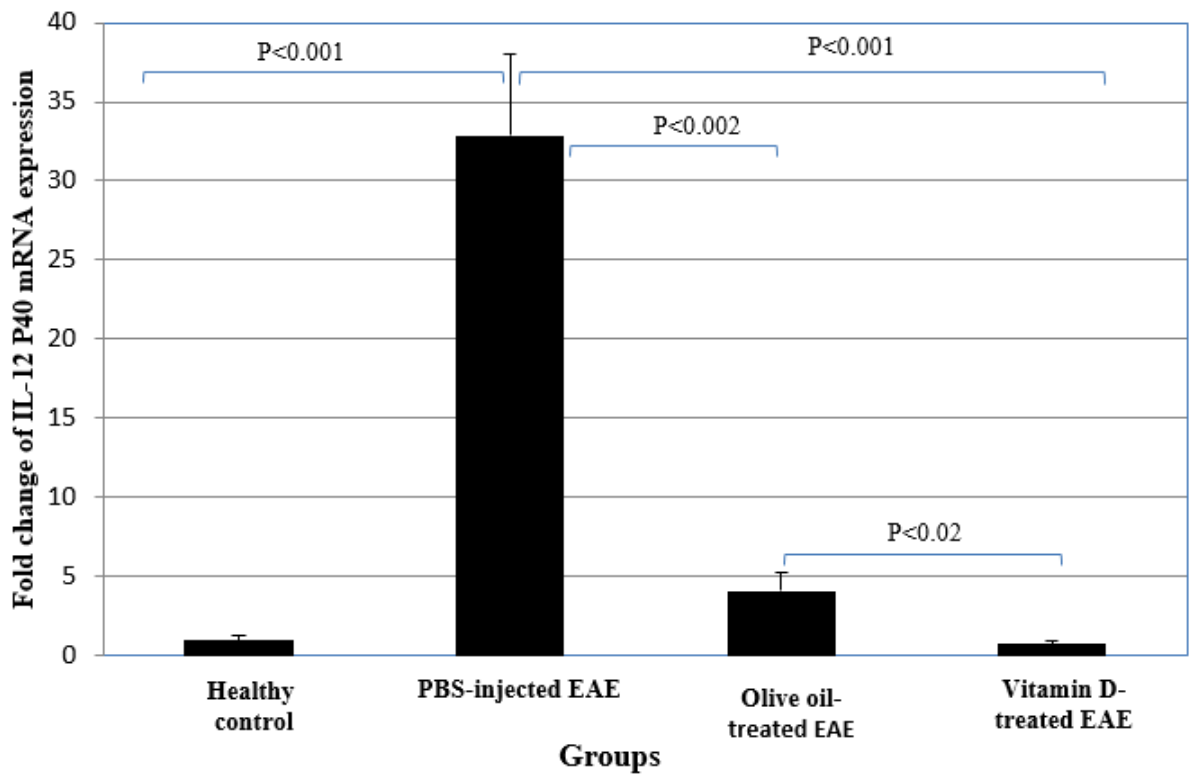

Fig 4. Comparison of the expression of IL-12 P40 mRNA between vitamin D-treated and control groups. The mRNA expression of the IL-12 P40 in PBS-treated EAE group was significantly higher than in the healthy group $(P<0.001$, by using student's $t$ test). The expression of IL-12 P40 mRNA in both vitamin D-treated EAE mice and olive oil-treated EAE group were decreased as compared with PBS-treated EAE group $(P<0.001$ and $P<0.002$, respectively; by using student's t test). The expression of IL-12 P40 mRNA in vitamin D-treated EAE group was also significantly lower than olive oil-treated EAE group $(P<0.02$, by using student's $t$ test). 
mRNA expression of IL-12 P35 in vitamin Dtreated EAE group was significantly higher than the healthy control group $(\mathrm{P}<0.05)$. However, there was no significant difference between EAE mice received vitamin $D$ treatment and the healthy group regarding IL-12 P40 expression (Table 2, Figures 3 \& 4).

The effect of vitamin D on the serum IL-12 levels

Serum IL-12 levels in PBS-injected EAE group and olive oil-administrated EAE group were significantly higher than healthy control mice $(\mathrm{P}<0.001$ and $\mathrm{P}<0.01$, respectively). Serum IL-12 levels in EAE group receiving vitamin $\mathrm{D}$ treatment were significantly diminished compared to PBSreceived and olive oil-administrated EAE mice $(\mathrm{P}<0.004$ and $\mathrm{P}<0.05$, respectively). Serum IL-12 levels were not significantly different between EAE group receiving vitamin $\mathrm{D}$ treatment and the healthy group mice (Table 2 and Figure 5).

The effect of vitamin $D$ on gene expression of TGF- $\beta$ in the spinal cords

There were no significant differences between PBS-injected EAE mice, olive oil-administrated EAE group and the healthy control mice regarding gene expression of the TGF- $\beta$. In vitamin D-treated EAE group, mRNA expression of TGF- $\beta$ was significantly increased compared to the healthy control group and EAE mice that received PBS $(\mathrm{P}<0.01$ and $\mathrm{P}<0.04$, respectively). TGF- $\beta$ expression was not significantly different between EAE group receiving vitamin D treatment and olive oil-administrated EAE mice, although this cytokine expression was found to be higher in vitamin Dtreated mice (Table 2 and Figure 6).

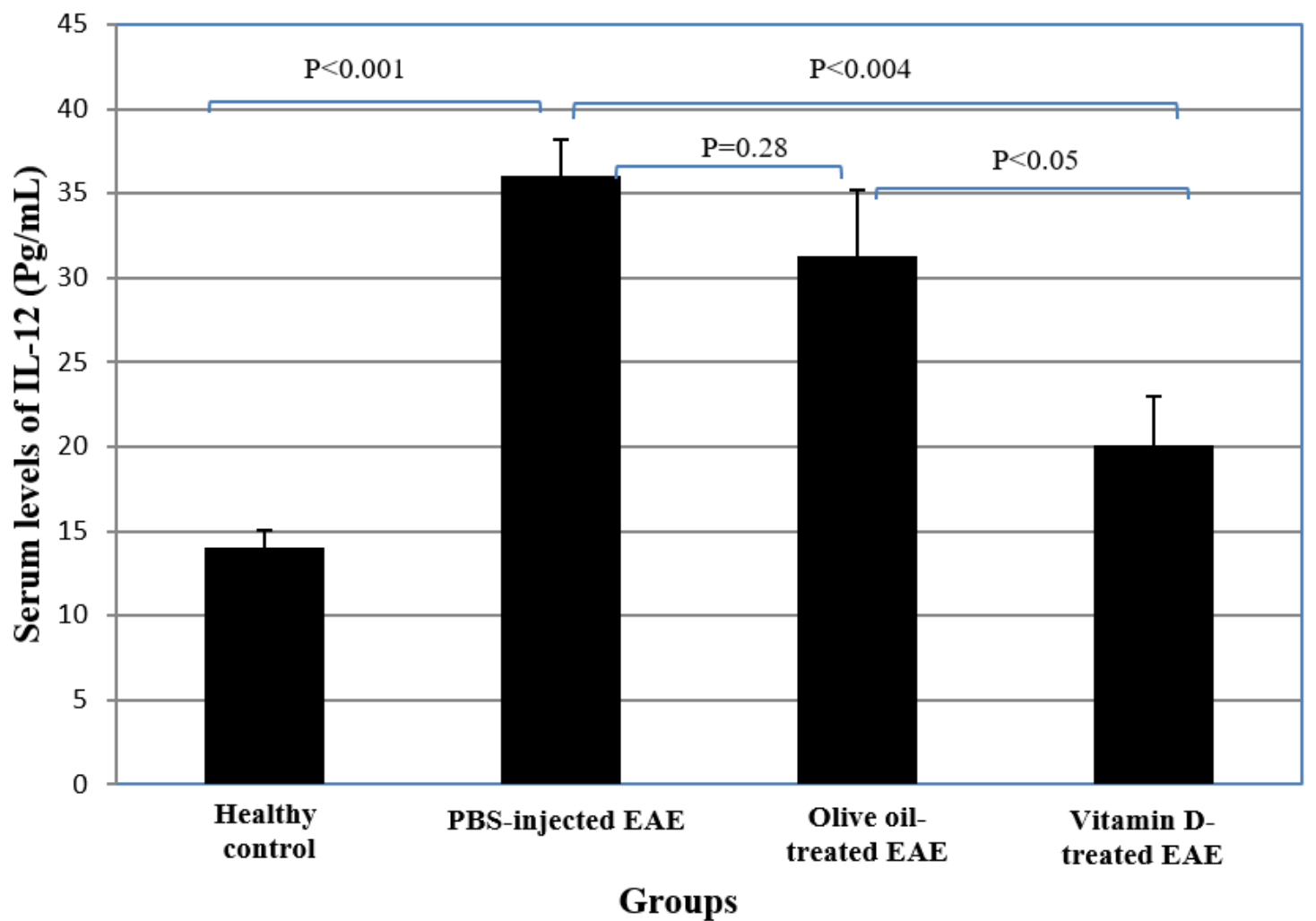

Fig 5. Comparison of the serum levels of IL-12 between vitamin D-treated and control groups.

The serum IL-12 levels of in PBS-injected and olive oil-treated EAE mice were significantly increased in comparison with to healthy control group ( $\mathrm{P}<0.001$ and $\mathrm{P}<0.01$, respectively; by using student's $t$ test). The serum levels of IL-12 in vitamin D-treated EAE group was significantly lower than in PBS-injected and olive oil-treated EAE mice $(\mathrm{P}<0.004$ and $\mathrm{P}<0.05$, respectively; by using student's $t$ test $)$. 


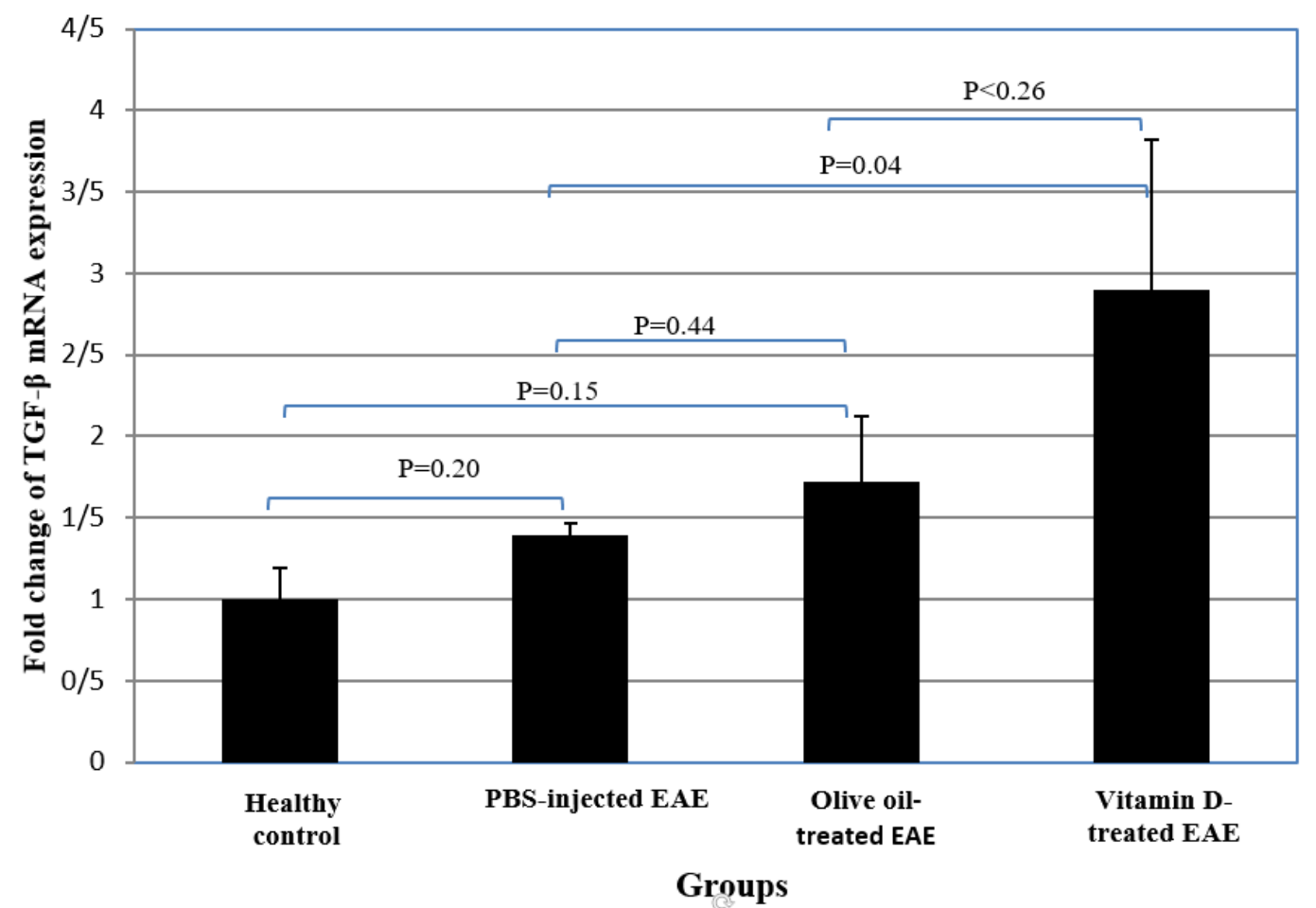

Fig 6. Comparisom of the expression of TGF- $\beta$ mRNA between ginger-treated and control groups.

No significant difference was observed between PBS-treated, olive oil-treated EAE mice an healthy control group groups regarding the expression of the TGF- $\beta$ mRNA. In vitamin D-treated EAE group the expression of TGF- $\beta$ mRNA was significantly higher as compared to healthy control group and PBS-treated EAE mice $(\mathrm{P}<0.01$ and 0.04 , respectively).

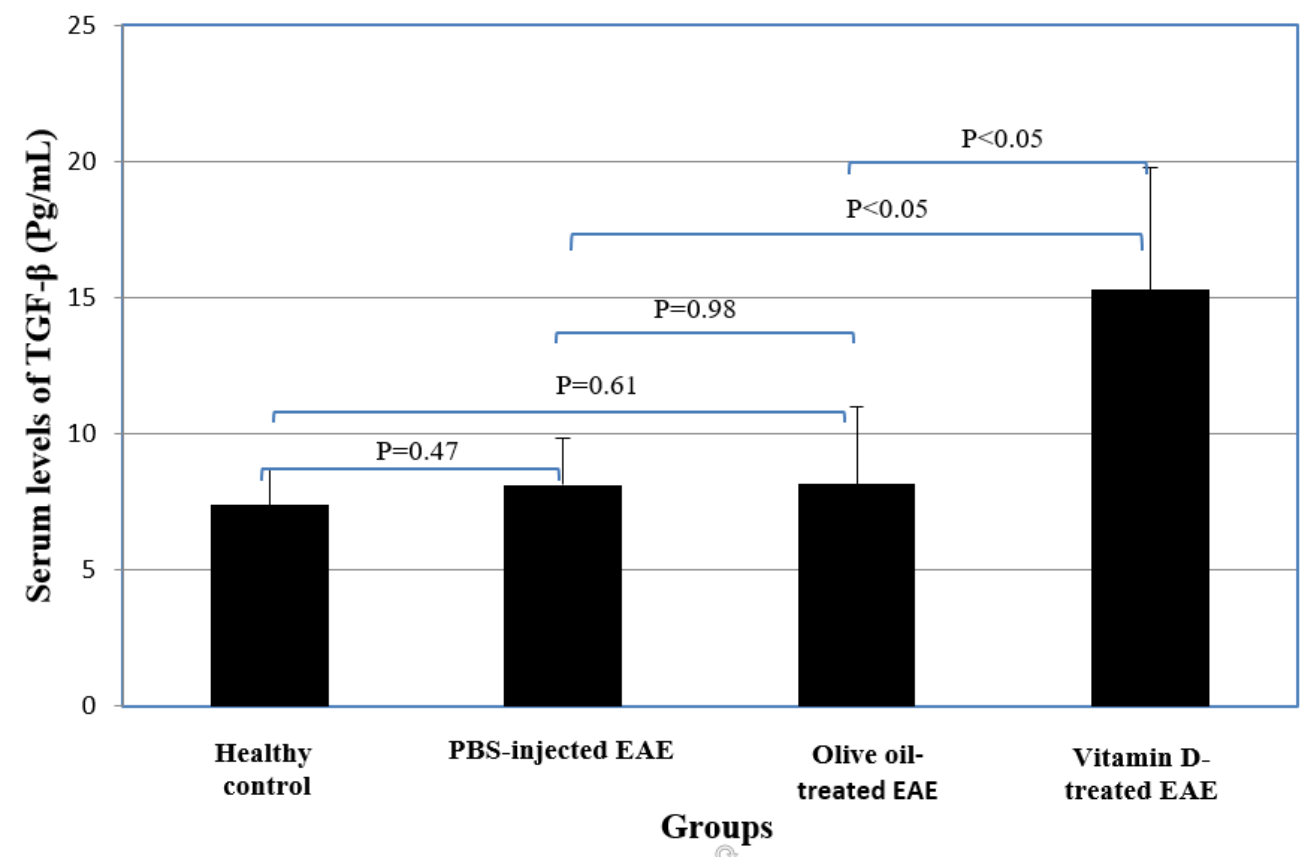

Fig 7. Comparisom of the expression of TGF- $\beta$ between vitamin-D and control groups.

No significant difference was observed between PBS-treated EAE mice, olive oil-treated EAE mice and healthy control group regarding the serum levels of TGF- $\beta$. In vitamin D-treated EAE group the mean serum TGF- $\beta$ was significantly higher than those in PBS-injected and olive oil-treated EAE mice ( $\mathrm{P}<0.05$, by using student's $t$ test). 


\section{The effect of vitamin $D$ on serum levels of TGF- $\beta$}

No significant differences were observed between PBS-injected EAE group, olive oiladministrated EAE mice and the healthy control group regarding serum TGF- $\beta$ levels. Vitamin Dtreated EAE group had significantly higher serum levels of TGF- $\beta$ than PBS-injected EAE group and olive oil- administrated EAE mice $(\mathrm{P}<0.05)$ (Table 2 and Figure 7).

\section{Discussion}

Our results indicated that clinical manifestations of EAE were decreased in vitamin D-treated EAE group compared to the control groups. Previous reports indicated an association between vitamin D deficiency and higher incidence and severity of MS (23-25). Furthermore, inverse correlations have been found between cerebral lesions, brain atrophy and the level of disability (26).

MS and EAE are Th1/Th17-mediated autoimmune diseases (4). IL-12 may play a major role in the development of MS and EAE by inducing differentiation of both Th1 and Th17 cells (8). Moreover, interferon- $\gamma$ [IFN- $\gamma$ (a main cytokine of Th1 cells)] may play a key role in the pathogenesis of EAE disease through increasing M1 macrophages expansion (27). Furthermore, IFN- $\gamma$ enhances the expression of inducible nitric oxide synthase (NOS) enzyme that lead to elevated NO synthesis by antigen presenting cells such as macrophages and DCs (28). Therefore, it seems that the IL-12/IFN- $\gamma / \mathrm{NO}$ axis plays a critical role in the development of EAE (28). Our results indicated elevated IL-12 expression in the spinal cords and serum from control EAE mice treated with PBS or olive oil. In agreement with our results, IL-12 production was increased by stimulated-peripheral blood mononuclear cells (PBMCs) from patients with MS (29). These observations confirm the possible contribution of IL-12 in MS and EAE development.

Our results also indicated that IL-12 expression decreased in the spinal cord and serum of EAE mice receiving vitamin D treatment. In accordance with our findings, vitamin D suppressed IL-12 secretion by lipopolysaccharides (LPS)-stimulated human microglial cells (30). Besides, vitamin D treatedsplenocytes from EAE mice produce higher levels of Th2 cell-related cytokines such as IL-10 (31). Moreover, it has been indicated that vitamin Dtreated-microglial cells express higher ratio of IL10/IL-12 (30). Additionally, vitamin D inhibits Th1 cells-type cytokines, while induces Th2 cells-type cytokines (32). Furthermore, TGF- $\beta$ has suppressive effects on the IL-12 synthesis by monocytes (16). Accordingly, some beneficial effects of vitamin D on EAE may act by suppression of IL-12 expression and induction inhibitory cytokines that may result in modulation of both Th1- and Th17 cells-related immune responses and deviation of responses from Th1- to Th2 cells.

Our results also indicated no significant difference between PBS-injected EAE mice and healthy control group regarding the expression of TGF- $\beta$. We have also previously reported that there was no significant difference between patients with MS and healthy subjects regarding circulating levels of a Treg-cells related cytokine IL-35 (33). In the present study, we measured TGF- $\beta$ expression on day 31 post immunization. However, it has been reported that serum levels of TGF- $\beta$ were diminished in the development stage of EAE (34). Therefore, reduced levels of TGF- $\beta$ may contribute in the EAE development during the early phase of disease.

Our results also indicated elevated TGF- $\beta$ expression in EAE mice receiving vitamin D treatment. In agreement with our findings, mRNA expression of TGF- $\beta$ and its serum levels were increased after vitamin D supplementation in patients with MS, while EDSS scores decreased $(35,36)$. TGF- $\beta$ is required for both Th17- and Treg cells differentiation. Stimulation of naive $T$ cells in the presence of TGF- $\beta$ (without IL-6) leads to the Treg cells differentiation, whereas, a combination of TGF- $\beta$ and IL- 6 results in Th17 cell differentiation (37). It seems that TGF- $\beta /$ IL- 6 ratio may be a determining factor in differentiating naïve $\mathrm{CD}^{+}$cells and Treg- or Th17 cells (38). The presence of higher levels of IL-6 together with adequate levels of TGF- $\beta$ may lead to differentiation of naïve Th cells into pathogenic 
Th17 cells, during initial stage of developing EAE $(34,39,40)$. We observed higher levels of TGF- $\beta$ in vitamin D-treated EAE group. Reducing effects of vitamin D on IL-6 production have been also demonstrated in other studies $(41,42)$. In the presence of the higher levels of TGF- $\beta$ and lower levels of IL- 6 (higher TGF- $\beta$ /IL- 6 ratio), TGF- $\beta$ leads to differentiation of protective Treg cells (38). Also, vitamin $\mathrm{D}$ alone or in joining with glucocorticoids, strongly stimulated the differentiation of Treg cells $(32,43)$. Therefore, treatment with vitamin D may improve the Th17/Treg cells imbalance with tendency toward Treg cells differentiation that may contribute an important role in the amelioration of EAE.

We also observed that mRNA expression of IL12 in olive oil-administrated group was significantly diminished compared to PBS-injected EAE group. These observations indicate that olive oil may have some anti-inflammatory effects alone. In agreement with our finding, anti-inflammatory effects of olive oil have been demonstrated in other studies $(44,45)$. Olive oil has been used as a vitamin D vehicle in our study. Accordingly, vitamin D and olive oil may have synergistic effects on detected parameters in our EAE model. Therefore, vitamin D and olive oil may have synergistically beneficial properties on EAE disease.

In summary, IL-12 expression was up-regulated in both spinal cord and serum of EAE mice. Therefore, increased IL-12 expression probably contributes in the EAE development. Vitamin D also ameliorates the severity of EAE and modulates IL-12 and TGF- $\beta$ expression in EAE mice. Further investigations should be conducted to consider the incorporation of vitamin D in the treatment of MS disease.

\section{Acknowledgments}

This work was supported by a grant from Rafsanjan University of Medical Sciences, Rafsanjan, Iran.

\section{Conflict of interest}

There was no conflict of interest.

\section{References:}

1. Milo R, Miller A. Revised diagnostic criteria of multiple sclerosis. Autoimmun Rev. 2014;9972(14):012.

\section{Robinson AP, Harp CT, Noronha A, Miller} SD. The experimental autoimmune encephalomyelitis (EAE) model of MS: utility for understanding disease pathophysiology and treatment. Handb Clin Neurol. 2014;122:173-89.

3. Raphael I, Nalawade S, Eagar TN, Forsthuber TG. T cell subsets and their signature cytokines in autoimmune and inflammatory diseases. Cytokine. 2015;74(1):5-17.

4. Ghaffari SA, Nemati M, Hajghani $H$, Ebrahimi H, Sheikhi A, Jafarzadeh A. Circulating concentrations of interleukin (IL)-17 in patients with multiple sclerosis: Evaluation of the effects of gender, treatment, disease patterns and IL-23 receptor gene polymorphisms. Iran $\mathrm{J}$ Neurol. 2017;16(1):15-25.

5. Jafarzadeh A, Mahdavi R, Jamali M, Hajghani H, Nemati M, Ebrahimi HA. Increased Concentrations of Interleukin-33 in the Serum and Cerebrospinal Fluid of Patients with Multiple Sclerosis. Oman Med J. 2016 Jan;31(1):40-5.

6. Jafarzadeh A, Ebrahimi HA, Bagherzadeh S, Zarkesh F, Iranmanesh F, Najafzadeh A, et al. Lower serum levels of Th2-related chemokine CCL22 in women patients with multiple sclerosis: a comparison between patients and healthy women. Inflammation. 2014;37(2):604-10.

\section{Jafarzadeh A, Bagherzadeh S, Ebrahimi} HA, Hajghani H, Bazrafshani MR, Khosravimashizi A, et al. Higher circulating levels of chemokine CCL20 in patients with multiple sclerosis: evaluation of the influences of chemokine gene polymorphism, gender, treatment and disease pattern. J Mol Neurosci. 2014;53(3):500-5.

8. Lasek W, Zagozdzon R, Jakobisiak M. Interleukin 12: still a promising candidate for tumor immunotherapy? Cancer Immunol Immunother. 2014;63(5):419-35. 
9. Teng MW, Bowman EP, McElwee JJ, Smyth MJ, Casanova JL, Cooper AM, et al. IL-12 and IL-23 cytokines: from discovery to targeted therapies for immune-mediated inflammatory diseases. Nat Med. 2015;21(7):719-29.

10. Happel KI, Dubin PJ, Zheng M, Ghilardi N, Lockhart C, Quinton LJ, et al. Divergent roles of IL-23 and IL-12 in host defense against Klebsiella pneumoniae. J Exp Med. 2005;202(6):761-9.

11. Jana M, Pahan K. IL-12 p40 homodimer, but not IL-12 p70, induces the expression of IL-16 in microglia and macrophages. Mol Immunol. 2009;46(5):773-83.

12. Cooper AM, Khader SA. IL-12p40: an inherently agonistic cytokine. Trends Immunol. 2007;28(1):33-8.

13. de Paus RA, Geilenkirchen MA, van Riet S, van Dissel JT, van de Vosse E. Differential expression and function of human IL-12Rbeta2 polymorphic variants. Mol Immunol. 2013;56(4):380-9.

14. Croxford AL, Kulig P, Becher B. IL-12and IL-23 in health and disease. Cytokine Growth Factor Rev. 2014;25(4):415-21.

15. Travis MA, Sheppard D. TGF-beta activation and function in immunity. Annu Rev Immunol. 2014;32:51-82.

16. Mantel PY, Schmidt-Weber CB. Transforming growth factor-beta: recent advances on its role in immune tolerance. Methods in molecular biology. 2011;677:303-38.

17. Mirshafiey A, Mohsenzadegan M. TGFbeta as a promising option in the treatment of multiple sclerosis. Neuropharmacol. 2009;56(67):929-36.

18. Mpandzou G, Ait Ben Haddou E, Regragui W, Benomar A, Yahyaoui M. Vitamin D deficiency and its role in neurological conditions: A review. Rev Neurol. 2016; 172(2):109-22.
19. Mohammadi-Kordkhayli M, AhangarParvin R, Azizi SV, Nemati M, Shamsizadeh A, Khaksari M, et al. Vitamin D Modulates the Expression of IL-27 and IL-33 in the Central Nervous System in Experimental Autoimmune Encephalomyelitis (EAE). Ir J Immunol. 2015;12(1):35-49.

20. Jafarzadeh A, Mohammadi-Kordkhayli M, Ahangar-Parvin R, Azizi V, Khoramdel-Azad $\mathrm{H}$, Shamsizadeh A, et al. Ginger extracts influence the expression of IL-27 and IL-33 in the central nervous system in experimental autoimmune encephalomyelitis and ameliorates the clinical symptoms of disease. J Neuroimmunol. 2014;276(1-2):80-8.

21. Takeuchi C, Yamagata K, Takemiya T. Variation in experimental autoimmune encephalomyelitis scores in a mouse model of multiple sclerosis. World J Neurol. 2013;3(3):5661.

22. Cantorna MT, Woodward WD, Hayes CE, DeLuca HF. 1,25-dihydroxyvitamin D3 is a positive regulator for the two anti-encephalitogenic cytokines TGF-beta 1 and IL-4. J Immunol. 1998;160(11):5314-9.

23. Ascherio A, Munger KL, Simon KC. Vitamin D and multiple sclerosis. Lancet Neurol. 2010;9(6):599-612.

24. Fitzgerald KC, Munger KL, Kochert K, Arnason BG, Comi G, Cook S, et al. Association of Vitamin D Levels With Multiple Sclerosis Activity and Progression in Patients Receiving Interferon Beta-1b. JAMA Neurol. 2015;72(12):1458-65.

25. Sundstrom P, Salzer J. Vitamin D and multiple sclerosis-from epidemiology to prevention. Acta Neurol Scand. 2015;132(199):5661.

26. Rolf L, Muris AH, Hupperts R, Damoiseaux J. Illuminating vitamin D effects on B cells - the multiple sclerosis perspective. Immunol. 2016;147(3):275-84. 
27. Dungan LS, McGuinness NC, Boon L, Lynch MA, Mills KH. Innate IFN-gamma promotes development of experimental autoimmune encephalomyelitis: A role for NK cells and M1 macrophages. Eur J Immunol. 2014;2014(23):201444612.

28. Xiao BG, Ma CG, Xu LY, Link H, Lu CZ. IL-12/IFN-gamma/NO axis plays critical role in development of Th1-mediated experimental autoimmune encephalomyelitis. Mol Immunol. 2008;45(4):1191-6.

29. Niino M, Fukazawa T, Miyazaki Y, Takahashi E, Minami N, Amino I, et al. Suppression of IL-10 production by calcitriol in patients with multiple sclerosis. J Neuroimmunol. 2014;270(1-2):86-94.

30. Verma R, Kim JY. 1,25Dihydroxyvitamin D3 Facilitates M2 Polarization and Upregulates TLR10 Expression on Human Microglial Cells. Neuroimmunomodulation. 2016; 23(2):75-80.

31. Waddell A, Zhao J, Cantorna MT. NKT cells can help mediate the protective effects of 1,25dihydroxyvitamin D3 in experimental autoimmune encephalomyelitis in mice. Int Immunol. 2015;27(5):237-44.

32. Hewison M. Vitamin D and the immune system: new perspectives on an old theme. Rheum Dis Clin North Am. 2012;38(1):125-39.

33. Jafarzadeh A, Jamali M, Mahdavi R, Ebrahimi HA, Hajghani H, Khosravimashizi A, et al. Circulating Levels of Interleukin-35 in Patients with Multiple Sclerosis: Evaluation of the Influences of FOXP3 Gene Polymorphism and Treatment Program. J Mol Neurosci. 2014;2014:19.

34. Lu P, Wang M, Zheng P, Hou J, Zhang Y, Deng Y, et al. Th17/Treg unbalance is involved in the pathogenesis of experimental autoimmune encephalomyelitis. Chin J Cell Mol Immunol. 2014;30(10):1013-7.
35. Shirvani-Farsani Z, Behmanesh M, Mohammadi SM, Naser Moghadasi A. Vitamin D levels in multiple sclerosis patients: Association with TGF-beta2, TGF-betaRI, and TGF-betaRII expression. Life Sci. 2015;134:63-7.

36. Aivo J, Hanninen A, Ilonen J, SoiluHanninen M. Vitamin D3 administration to MS patients leads to increased serum levels of latency activated peptide (LAP) of TGF-beta. J Neuroimmunol. 2015;280:12-5.

37. Zhang $\mathrm{C}$, Zhang $\mathrm{X}$, Chen XH. Inhibition of the interleukin- 6 signaling pathway: a strategy to induce immune tolerance. Clin Rev Allergy Immunol. 2014;47(2):163-73.

38. Etesam Z, Nemati M, Ebrahimizadeh MA, Ebrahimi HA, Hajghani H, Khalili T, Frootan R, Zinoddini N, Jafarzadeh A. Altered Expression of Specific Transcription Factors of Th17 (RORgammat, RORalpha) and Treg Lymphocytes (FOXP3) by Peripheral Blood Mononuclear Cells from Patients with Multiple Sclerosis. J Mol Neurosci. 2016; 60(1):94-101.

39. Murphy AC, Lalor SJ, Lynch MA, Mills KH. Infiltration of Th1 and Th17 cells and activation of microglia in the CNS during the course of experimental autoimmune encephalomyelitis. Brain Behav Immun. 2010;24(4):641-51.

40. Ivanova EA, Orekhov AN. T Helper Lymphocyte Subsets and Plasticity in Autoimmunity and Cancer: An Overview. BioMed Res Int. 2015;2015:327470.

41. Xu QA, Li ZF, Zhang P, Cao LH, Fan MW. Effects of 1,25-dihydroxyvitamin D on Macrophage Cytokine Secretion Stimulated by Porphyromonas gingivalis. Jpn J Infect Dis. 2016.

42. Huang YN, Ho YJ, Lai CC, Chiu CT, Wang JY. 1,25-Dihydroxyvitamin D3 attenuates endotoxin-induced production of inflammatory mediators by inhibiting MAPK activation in primary cortical neuron-glia cultures. J Neuroinflammation. 2015;12:147. 
43. Barrat FJ, Cua DJ, Boonstra A, Richards DF, Crain C, Savelkoul HF, et al. In vitro generation of interleukin 10-producing regulatory $\mathrm{CD} 4+\mathrm{T}$ cells is induced by immunosuppressive drugs and inhibited by $\mathrm{T}$ helper type 1 (Th1)-and Th2inducing cytokines. The Journal of experimental medicine. 2002;195(5):603-16.
44. Takashima T, Sakata Y, Iwakiri R, Shiraishi R, Oda Y, Inoue N, et al. Feeding with olive oil attenuates inflammation in dextran sulfate sodium-induced colitis in rat. J Nutr Biochem. 2014;25(2):186-92.

45. Rodriguez-Morato J, Xicota L, Fito M, Farre M, Dierssen M, de la Torre R. Potential role of olive oil phenolic compounds in the prevention of neurodegenerative diseases. Molecules. 2015;20(3):4655-80.

\section{How to Cite This Article}

Ahangar-Parvin R, Mohammadi-Kordkhayli M, Azizi S, Nemati M, Khorramdel-Azad H, Taghipour Z, et al. The Modulatory Effects of Vitamin D on the Expression of IL-12 and TGF- $\beta$ in the Spinal Cord and Serum of Mice with Experimental Autoimmune Encephalomyelitis. Iran J Pathol, 2018; 13(1): 10-22. 